\title{
DERIVATIVES AND LEBESGUE POINTS VIA HOMEOMORPHIC CHANGES OF SCALE
}

BY

DON L. HANCOCK

\begin{abstract}
Let $I$ be a closed interval, and suppose $\mathscr{K}, \mathcal{K}$, and $\Lambda$ denote, respectively, the class of homeomorphisms of $I$ onto itself, the class of homeomorphisms of the line onto itself, and the class of real functions on $I$ for which each point is a Lebesgue point. Maximoff proved that $\Lambda \circ \mathcal{K}$ is exactly the class of Darboux Baire 1 functions, where $\Lambda \circ \mathcal{K}=\{f \circ k: f \in \Lambda, k \in \mathcal{K}\}$. The present paper is devoted primarily to a study of $\mathcal{X} \circ \Lambda=\{h \circ f: f \in \Lambda, h \in \mathcal{X}\}$. The characterizations of this class which are obtained show that a function is a member of $\mathcal{X} \circ \Lambda$ if and only if, in addition to the obvious requirement of approximate continuity, it satisfies certain growth and density-like conditions. In particular, any approximately continuous function with countably many non-Lebesgue points belongs to $\mathcal{H} \circ \Lambda$. It is also established that $\mathcal{H} \circ \Lambda$ is a uniformly closed algebra properly containing the smallest algebra generated from $\Lambda$, and a characterization of the latter algebra is provided.
\end{abstract}

1. Introduction. Many familiar properties of real functions, defined on a closed interval $[a, b]$, can be neither created nor destroyed by a homeomorphic change of variables or a homeomorphic change of scale of the " $y$-axis." However, a variety of important properties-especially of some classes of functions linked closely with the study of differentiation-are not invariant under such homeomorphic transformations. For example, differentiability is not an invariant characteristic, since any continuous function of bounded variation can be transformed into a differentiable function by a suitable homeomorphic change of variables [6]. Likewise, the property of being a derivative is not invariant, because any bounded approximately discontinuous derivative ceases to be a derivative under outer composition with any strictly convex homeomorphism [5].

Suppose that $\mathscr{F}$ is a class of real functions defined on $[a, b]$, and that $\mathscr{K}$ and $\mathcal{H}$ denote, respectively, the class of homeomorphisms of $[a, b]$ onto itself and the class of homeomorphisms of the line onto itself. Imitating notation adopted by Bruckner in [4], let $\mathscr{F} \circ \mathcal{K}=\{f \circ k: f \in \mathscr{F}, k \in \mathscr{K}\}$ and $\mathcal{H} \circ \mathscr{F}=\{h \circ f: h \in \mathcal{K}, f \in \mathscr{F}\}$. These latter classes arise naturally in investigations of the effect of homeomorphic changes of scales on the class $\mathcal{F}$. Observe that if $\mathcal{G}=\mathcal{H} \circ \mathcal{F}$, then $\mathcal{H} \circ \mathcal{G}=\mathcal{F}$; hence, $\mathcal{H} \circ \mathscr{F}$ consists precisely of those functions which can be transformed into $\mathcal{F}$ via outer composition with a member of $\mathcal{H}$. An analogous statement is, of course, also valid for $\mathscr{F} \circ \mathcal{K}$. Several authors have made a number of studies lately characterizing the classes $\mathcal{H} \circ \mathscr{F}$ and $\mathscr{F} \circ \mathcal{K}$, particularly for various classes $\mathscr{F}$

Received by the editors October 24, 1980.

1980 Mathematics Subject Classification. Primary 26A24; Secondary 26A99.

(C) 1981 American Mathematical Society

$0002-9947 / 81 / 0000-0413 / \$ 06.50$ 
related to differentiation theory. (For instance, Fleissner and Foran [7] recently characterized $\mathscr{H} \circ \mathscr{F}$ and $\mathscr{F} \circ \mathscr{K}$ when $\mathscr{F}$ is the class of differentiable functions, while Bruckner [3] characterized $\mathscr{F} \circ \mathscr{K}$ and Bari [1] characterized $\mathcal{H} \circ \mathscr{F}$ when $\mathscr{F}$ is the class of differentiable a.e. functions.) In [4, Chapter 9], and in the expository article [2], a summary of results along these lines may be found, together with a discussion of some applications and unsolved problems. A particularly striking theorem was advanced by Maximoff in a series of deep papers [10]-[12]. In an attempt to shed light on the structure of derivatives, Maximoff showed that if $\Lambda$ denotes the class of functions for which each point is a Lebesgue point, then $\Lambda \circ \mathscr{K}$ is the class of Darboux Baire 1 functions. (Maximoff's original proof was actually incorrect, but Preiss [14] has recently provided a valid proof.)

The major purpose of this paper is to study the class $\mathcal{H} \circ \Lambda$, henceforth denoted simply by $C A^{*}$. Obviously $C A^{*} \subset C A$, where $C A$ represents the class of approximately continuous functions, because $\Lambda \subset C A$ and approximate continuity is invariant under outer composition with continuous functions. The task of characterizing $C A^{*}$ thus reduces to the problem of determining necessary and sufficient conditions under which an approximately continuous function can be transformed into $\Lambda$ via outer composition with some homeomorphism. $\$ 4$ contains the main results of the paper, including two characterizations of $C A^{*}$. Although explicit statements of the theorems are deferred until the necessary technical machinery has been introduced, we may summarize them by saying that an approximately continuous function is transformable into $\Lambda$ if and only if it satisfies certain growth and density-like conditions. We exploit these results in our constructions of functions in $C A \sim C A^{*}$. Then, in $\S 5$, we establish that $C A^{*}$ is a uniformly closed algebra properly containing the smallest algebra generated from $\Lambda$. Moreover, we show that in a certain sense the former algebra is vast in comparison to the latter one.

In the process of obtaining the results mentioned above, we obtain several others. In particular, if $S$ is a subset of the set of non-Lebesgue points of an approximately continuous function $f$, then we provide in $\$ 3$ some conditions on $S$ which are necessary for the existence of a homeomorphism $h \in \mathcal{H}$ such that every point of $S$ is a Lebesgue point of $h \circ f$.

The work in this paper was based on a portion of the author's doctoral dissertation. The author gratefully acknowledges the supervision of Professor Andrew Bruckner of the University of California, Santa Barbara.

2. Preliminaries. In this section we specify the notation and terminology necessary to our discussion, and set down a few background facts to be used in the sequel.

The real line is denoted by $R$ and $[a, b]$ is an arbitrary nondegenerate closed interval. For any set $A \subset[a, b], \mathrm{Cl}(A)$ stands for the closure of $A$ and $A^{c}$ for the complement of $A$ relative to $[a, b]$. The letters $m$ and $n$ always denote positive integers. All functions are considered to be finite and real valued. Unless explicitly stated otherwise, the homeomorphisms we consider are from $R$ onto $R$, while we assume that all other functions $f$ are (Lebesgue) measurable with domain $[a, b]$. 
Lebesgue measure is denoted by $\lambda$, all integrals are to be interpreted as Lebesgue integrals, and we set $\int_{x}^{y} f=\int_{y}^{x} f=\int_{[x, y]} f$. For any constant $T$, we use the notation $\{f>T\}$ in lieu of $\{t: f(t)>T\}$. If $E$ is a subset of $[a, b], f \mid E$ denotes the restriction of $f$ to $E$. Given a point $x$ and a measurable set $A$, we use $\bar{d}^{+}(A, x)$ to symbolize the upper right density of $A$ at $x$; i.e.,

$$
\bar{d}^{+}(A, x)=\varlimsup_{y \rightarrow x^{+}} \frac{\lambda(A \cap(x, y))}{y-x} .
$$

Analogously, $\bar{d}^{-}(A, x)$ and $\bar{d}(A, x)$ symbolize the upper left and upper densities of $A$ at $x$, respectively, while the density of $A$ at $x$ (when it exists) is symbolized by $d(A, x)$. The set consisting of all the Lebesgue points of $f$ is called the Lebesgue set of $f$ and is denoted by $L_{f}$; hence, $x \in L_{f}$ if and only if

$$
\lim _{y \rightarrow x} \frac{1}{y-x} \int_{x}^{y}|f(t)-f(x)| d t=0 .
$$

We use $\Lambda, C A^{*}$, and $C A$ as specified in the introduction, and we use $\Delta$ to denote the class of derivatives on $[a, b]$.

Fundamental properties of functions in the classes $C A, \Delta$, and $\Lambda$ may be found in [4], and we shall use these properties freely. It is easily verified that at each $x \in L_{f}, f$ is approximately continuous and is the derivative of its integral; hence, $\Lambda \subset C A$ and $\Lambda \subset \Delta$. Additionally, if $f$ is bounded, then $f \in C A$ implies $f \in \Lambda$. The last fact is a well-known consequence of the following lemma.

LEMMA 2.1. Suppose that $f$ is approximately continuous at $x$ and $A$ is a measurable subset of $[a, b]$. If $f \mid A$ is bounded, then

$$
\lim _{y \rightarrow x} \frac{1}{y-x} \int_{(x, y) \cap A}|f(t)-f(x)| d t=0 .
$$

If, in addition, $d(A, x)=1$, then

$$
\lim _{y \rightarrow x} \frac{1}{|y-x|} \int_{(x, y) \cap A}|f(t)| d t=|f(x)| .
$$

A proof of Lemma 2.1 may be found in [4]. The next lemma, which may be easily established using the previous lemma, will facilitate our later work.

LEMMA 2.2. Let $f$ be approximately continuous at $x$ and let $B=\{|f|>|f(x)|+c\}$, where $c$ is a positive constant. Then $x \in L_{f}$ if and only if

$$
\lim _{y \rightarrow x} \frac{1}{y-x} \int_{(x, y) \cap B}|f(t)| d t=0 .
$$

We conclude this section by mentioning that it is easy to verify that any approximately continuous derivative bounded from above or below must belong to $\Lambda$. The proof relies on Lemma 2.2 and the fact that a summable derivative must be the derivative of its integral.

3. Creating Lebesgue points. We shall establish in $\$ 4$ that not every approximately continuous function can be transformed into $\Lambda$ by a homeomorphic change of scale of the function's range. Nonetheless, in this section we show that if $f$ is a 
member of $C A \sim \Lambda$ and $S$ is a countable subset of $L_{f}^{c}$, then there exists some homeomorphism $h \in \mathcal{K}$ such that $S \cup L_{f} \subset L_{h \circ f}$. In the course of demonstrating this fact, we obtain several results which will be utilized again in $\$ 4$ to help establish our main theorems.

Before we focus our attention on the task of "creating" Lebesgue points via homeomorphic changes of scale, we find it desirable to comment on the structure of $L_{f}^{c}$ when $f \in C A$. Since every approximately continuous function is Baire $1, f$ must have a dense set of points of continuity in $[a, b]$. About any point $x$ at which $f$ is continuous, there exists an open interval $N_{x}$ on which $f$ must be bounded. It follows from Lemma 2.1 that each point of $N_{x}$ is a Lebesgue point; hence, $L_{f}$ contains a dense open subset of $[a, b]$. This demonstrates that $L_{f}^{c}$ must be nowhere dense. Moreover, provided that $f$ is also summable, it is shown in [8] that $L_{f}^{c}$ is a Borel nullset of at most type $G_{\delta \sigma \delta \sigma}$. Summable approximately continuous functions with uncountable non-Lebesgue sets are easily constructed, as Example 3.1 below illustrates.

EXAMPLE 3.1. Let $\left\{I_{m}\right\}=\left\{\left(a_{m}, b_{m}\right)\right\}$ be an enumeration of the open intervals contiguous to a nowhere dense perfect nullset $P \subset[a, b]$. For each $m$, let $\left(\alpha_{m}, \beta_{m}\right)$ be the subinterval of $I_{m}$ having length $\lambda\left(I_{m}\right) / m$ and midpoint $\frac{1}{2}\left(a_{m}+b_{m}\right)$. Define a function $f$ as follows: $f(x)=2 m$ if $x=\frac{1}{2}\left(a_{m}+b_{m}\right), f$ is identically 0 on the complement of $\cup_{m=1}^{\infty}\left(\alpha_{m}, \beta_{m}\right)$, and $f$ is defined elsewhere by linear extension. Observe that $\int_{I_{m}} f=\lambda\left(I_{m}\right)$, so $f$ is summable. Furthermore, straightforward calculations show that each point of $P$ is a point of approximate continuity but not a Lebesgue point. Since $f$ is continuous at every point of $P^{c}$, we conclude that $f \in C A$ and $L_{f}^{c}=P$.

If $f$ is a given function in $C A$, then for each $x \in[a, b]$ let $c_{x}$ be the extended real number defined by

$$
c_{x}=\varlimsup_{y \rightarrow x} \frac{1}{|y-x|} \int_{x}^{y}|f(t)-f(x)| d t .
$$

It follows that $x \in L_{f}^{c}$ if and only if $c_{x}$ is a positive number or $\infty$. We wish to distinguish those non-Lebesgue points for which $c_{x}$ is finite from those for which $c_{x}$ is infinite.

Definition 3.2. A point $x \in L_{f}^{c}$ is called weak if $0<c_{x}<\infty$, or it is called firm if $c_{x}=\infty$. Let $\left\{x: 0<c_{x}<\infty\right\}$ be symbolized by $W_{f}$.

For future use, we remark that it is not difficult to verify that $x$ is a firm non-Lebesgue point of $f$ if and only if

$$
\varlimsup_{y \rightarrow x} \frac{1}{|y-x|} \int_{x}^{y}|f(t)| d t=\infty .
$$

The "spike" function constructed in Example 3.1 has the property that every non-Lebesgue point is weak, since crude estimates show that $c_{x}<2$ for each $x \in P$. However, by suitably increasing the heights of the "spikes" we may obtain a summable approximately continuous function with uncountably many firm non-Lebesgue points. In [8] it is shown that $L_{f}^{c} \sim W_{f}$ is a Borel set of at most type $F_{\sigma \delta}$, if $f$ is any summable member of $C A$. 
Let us now introduce the following suggestive language: a homeomorphism $h \in \mathscr{H}$ creates (preserves) a Lebesgue point at $x \in L_{f}^{c}\left(x \in L_{f}\right)$ if $x \in L_{h_{\circ} f}$. Extending earlier notation, if $S$ is a subset of $[a, b]$, we define

$$
C A_{S}^{*}=\left\{f \in C A: S \subset L_{h \circ f} \text { for some } h \in \mathcal{H}\right\} .
$$

(If $S=[a, b], C A_{S}^{*}=C A^{*}$.) In this section we shall determine some necessary conditions under which a function is a member of $C A_{S}^{*}$.

Whenever investigating whether or not a function $f$ belongs to $C A_{s}^{*}$, we may make two assumptions which simplify the discussion. First, we may assume that only increasing homeomorphisms mapping 0 onto itself need be considered as our vehicles for creating Lebesgue points. For if $h_{1} \in \mathcal{H}$, then there exists an increasing homeomorphism $h \in \mathcal{H}$ such that $h(0)=0$ and $|h(u)-h(v)|=\left|h_{1}(u)-h_{1}(v)\right|$ for each $u$ and $v$ in $R$; hence, $L_{h \circ f}=L_{h_{1} \circ f}$. Accordingly, in the sequel we abandon our present usage of $\mathcal{H}$ and agree instead to use $\mathcal{H}$ exclusively to denote the set of all increasing homeomorphisms of $R$ onto $R$ which map 0 onto itself. Second, our next lemma clearly implies that we may assume the homeomorphisms we apply to $f$ preserve the Lebesgue points of $f$.

LeMma 3.3. Let $h$ and $H$ belong to $\mathcal{H}$. If $f \in C A$ and there exists a number $j$ such that $|h(u)| \leqslant|H(u)|$ whenever $|u| \geqslant j$, then $L_{H \circ f} \subset L_{h \circ f}$.

Proof. Suppose that $x$ is an arbitrary point in $L_{H} \circ f$. Choose

$$
\gamma=\max (H(j),|H(-j)|,|h(f(x))|+|H(f(x))|+1),
$$

and let $B=\{|h \circ f|>\gamma\}$ and $B_{1}=\{|H \circ f|>\gamma\}$. It is evident that $|h(f(t))|<$ $|H(f(t))|$ whenever $t \in B$. This implies that $B \subset B_{1}$ and

$$
\frac{1}{|y-x|} \int_{(x, y) \cap B}|h(f(t))| d t \leqslant \frac{1}{|y-x|} \int_{(x, y) \cap B_{1}}|H(f(t))| d t .
$$

Since $x \in L_{H} \circ f$, Lemma 2.2 implies that the right expression tends to 0 as $y$ approaches $x$. Applying Lemma 2.2 to $h \circ f$ completes the proof.

By definition, $f \in C A_{S}^{*}$ means $S \subset L_{h \circ f}$ for some $h \in \mathcal{H}$. Since there is no homeomorphism in $\mathcal{H}$ whose growth rate is minimum, Lemma 3.3 permits us to choose $h$ so that it possesses various properties. For example, we shall occasionally find it convenient to choose $h$ so that $\lim _{|u| \rightarrow \infty}|h(u) / u|=0$, or to choose $h$ so that it is odd.

Our first theorem below suggests why the terminology adopted in Definition 3.2 is appropriate: weak non-Lebesgue points are "weak" in the sense that any homeomorphism which meets a mild growth condition will create a Lebesgue point at each point of $W_{f}$ for every $f \in C A$.

THEOREM 3.4. Suppose that $h$ is any member of $\mathcal{H}$ with the property that $\lim _{|u| \rightarrow \infty}|h(u) / u|=0$. Then every point of approximate contimuity in $W_{f} \cup L_{f}$ belongs to $L_{h \circ f}$.

Proof. Suppose $x$ is a point of approximate continuity in $W_{f} \cup L_{f}$. Then

$$
\varlimsup_{y \rightarrow x} \frac{1}{|y-x|} \int_{x}^{y}|f(t)-f(x)| d t=c<\infty .
$$


Fix $\varepsilon>0$. Since $h \in \mathcal{K}$ and $\lim _{|u| \rightarrow \infty}|h(u) / u|=0$, there exists a large enough integer $N$ such that

$$
|h(u)-h(f(x))|<\frac{\varepsilon}{2(c+1)}|u-f(x)|, \quad \text { whenever }|u|>N .
$$

Let $A=\{|f| \leqslant N\}$ and $B=A^{c}$. By (1), and by Lemma 2.1 applied to $h \circ f$, we may choose $\delta>0$ such that $0<|y-x|<\delta$ implies

$$
\frac{1}{|y-x|} \int_{(x, y) \cap A}|h(f(t))-h(f(x))| d t<\frac{\varepsilon}{2}
$$

and

$$
\frac{1}{|y-x|} \int_{x}^{y}|f(t)-f(x)| d t<c+1 .
$$

It follows that if $0<|y-x|<\delta$, then

$$
\begin{aligned}
\frac{1}{|y-x|} \int_{x}^{y} \mid & h(f(t))-h(f(x))\left|d t<\frac{\varepsilon}{2}+\frac{1}{|y-x|} \int_{(x, y) \cap B}\right| h(f(t))-h(f(x)) \mid d t \\
& <\frac{\varepsilon}{2}+\frac{\varepsilon}{2(c+1)} \frac{1}{|y-x|} \int_{(x, y) \cap B}|f(t)-f(x)| d t \quad(\text { by }(2)) \\
& <\frac{\varepsilon}{2}+\frac{\varepsilon}{2(c+1)}(c+1)=\varepsilon .
\end{aligned}
$$

Therefore, $x \in L_{h \circ f}$. This completes the proof.

Since every non-Lebesgue point of the function constructed in Example 3.1 is weak, Theorem 3.4 indicates that the function belongs to $C A^{*}$. Even if $x$ is a firm non-Lebesgue point of some $f \in C A$, the next result shows that a homeomorphism can be found which creates a Lebesgue point at least at $x$.

THEOREM 3.5. If $f$ is approximately continuous at $x$, then there exists a homeomorphism $h \in \mathcal{H}$ such that $x \in L_{h \circ f}$.

Proof. Let $F=|f|$, where $f$ is approximately continuous at $x$. We first establish that to each $\alpha>0$ there corresponds a $T_{\alpha}>0$ such that

$$
\lambda\left(I \cap\left\{F \geqslant T_{\alpha}\right\}\right) \leqslant \alpha \lambda(I)
$$

whenever $I$ is any subinterval of $[a, b]$ which contains $x$. If $E=\{F>F(x)+1\}$, then approximate continuity implies that $d(E, x)=0$. Thus, there exists $\delta>0$ such that $\lambda(I \cap E) / \lambda(I) \leqslant \alpha$ whenever $x \in I \subset[a, b]$ and $\lambda(I)<\delta$. Since $f$ is finite, we may obtain a number $T_{\alpha}>F(x)+1$ for which $\lambda\left(\left\{F \geqslant T_{\alpha}\right\}\right)<\alpha \delta$. Hence, $\lambda(I)<\delta$ implies that $\lambda\left(I \cap\left\{F \geqslant T_{\alpha}\right\}\right) \leqslant \lambda(I \cap E) \leqslant \alpha \lambda(I)$, while $\lambda(I) \geqslant \delta$ implies that $\lambda\left(I \cap\left\{F \geqslant T_{\alpha}\right\}\right) \leqslant \alpha \delta \leqslant \alpha \lambda(I)$.

From (3), it obviously follows that for each $n$ there exists a positive number $T_{n}$ such that $\lambda\left((x, y) \cap\left\{F \geqslant T_{n}\right\}\right) \leqslant|y-x| / n^{3}$ for all $y \neq x$. Without loss of generality, assume these $T_{n}$ are chosen so that $T_{n+1}>T_{n}$ and $T_{n} \rightarrow \infty$. Let $H$ be any odd homeomorphism in $\mathcal{H}$ with the property that $H\left(T_{n}\right) \leqslant n-1$ for all $n>1$. 
Then, for all $y \neq x$ we have

$$
\begin{aligned}
\frac{1}{|y-x|} \int_{x}^{y}|H(f(t))| d t= & \frac{1}{|y-x|} \int_{x}^{y} H(F(t)) d t \\
\leqslant & \frac{H\left(T_{1}\right) \lambda\left((x, y) \cap\left\{0 \leqslant F<T_{1}\right\}\right)}{|y-x|} \\
& +\sum_{n=2}^{\infty} H\left(T_{n}\right) \frac{\lambda\left((x, y) \cap\left\{T_{n-1} \leqslant F<T_{n}\right\}\right)}{|y-x|} \\
\leqslant & H\left(T_{1}\right)+\sum_{n=2}^{\infty}(n-1) \frac{\lambda\left((x, y) \cap\left\{F>T_{n-1}\right\}\right)}{|y-x|} \\
\leqslant & H\left(T_{1}\right)+\sum_{n=2}^{\infty}(n-1) \frac{1}{(n-1)^{3}} \\
& <H\left(T_{1}\right)+2 .
\end{aligned}
$$

Therefore,

$$
\varlimsup_{y \rightarrow x} \frac{1}{|y-x|} \int_{x}^{y}|H(f(t))| d t<\infty .
$$

From the remark we made after Definition 3.2, it follows that $x$ is not a firm non-Lebesgue point of $H \circ f$. Hence, by Theorem 3.4 there exists an $H_{1} \in \mathcal{H}$ such that $x \in L_{H_{1} \circ H \circ f}$. Taking $h$ to be $H_{1} \circ H$ completes the proof.

In conjunction with Lemma 3.3 and Theorem 3.5, the following lemma implies that if $f \in C A$ and $S$ is any countable subset of $L_{f}^{c}$, then there exists some homeomorphism which creates a Lebesgue point at each point of $S$.

LEMMA 3.6. Let $\left\{h_{n}\right\}$ be any sequence of homeomorphisms in $\mathcal{H}$. Then there exists an odd homeomorphism $h \in \mathcal{H}$ such that for every $n$ there is a number $j_{n}$ for which $|u| \geqslant j_{n}$ implies $|h(u)| \leqslant\left|h_{n}(u)\right|$.

Proof. For each $n$, define a function $H_{n}$ by

$$
H_{n}(u)=\left\{\begin{array}{l}
\min \left(h_{1}(u),\left|h_{1}(-u)\right|, \ldots, h_{n}(u),\left|h_{n}(-u)\right|\right) \text { for } u>0 \\
-H_{n}(-u) \text { for } u<0
\end{array}\right.
$$

Clearly, $H_{n}$ must be an odd homeomorphism in $\mathcal{K}$, and $\left|h_{n}(u)\right|>\left|H_{n}(u)\right|>$ $\left|H_{n+1}(u)\right|$ for all $u$. Since each $H_{n}$ is increasing and unbounded, there exists a strictly increasing sequence $\left\{j_{n}\right\}$ of positive numbers with the property that $H_{n}\left(j_{n}\right)=n$ for every $n$. We now define a new homeomorphism $H \in \mathcal{H}$ as follows: $H(0)=0, H\left(j_{n}\right)=n, H\left(-j_{n}\right)=-n$, and $H$ is defined elsewhere on $R$ by linear extension. Finally, let $h$ be defined by

$$
h(u)=\left\{\begin{array}{l}
H(u), \quad \text { for } u \in\left[0, j_{1}\right] \text { and for } u=j_{2}, j_{3}, \ldots \\
\min \left(H(u), H_{n}(u)\right) \text { for } u \in\left(j_{n}, j_{n+1}\right), n=1,2, \ldots \\
-h(-u) \text { for } u<0
\end{array}\right.
$$


It is easy to verify that $h$ is an odd homeomorphism in $\mathcal{H}$. Moreover, it is clear from our definition of $h$ that $|h(u)| \leqslant\left|H_{n}(u)\right|$ whenever $|u| \geqslant j_{n}$. Hence $|h(u)| \leqslant$ $\left|h_{n}(u)\right|$ for all $|u| \geqslant j_{n}$.

THEOREM 3.7. Let $S=\cup_{n=1}^{\infty} S_{n}$, where $S_{n} \subset[a, b]$. If $f \in C A_{S_{n}}^{*}$ for each $n$, then $f \in C A_{S}^{*}$.

Proof. Suppose $f \in C A_{S_{n}}^{*}$ for each $n$. Then there exists a sequence $\left\{h_{n}\right\}$ of homeomorphisms in $\mathcal{H}$ such that $S_{n} \subset L_{h_{n} \circ f}$. Lemma 3.6 guarantees the existence of an $h \in \mathcal{H}$ and a sequence of numbers $\left\{j_{n}\right\}$ such that $|h(u)| \leqslant\left|h_{n}(u)\right|$ whenever $|u| \geqslant j_{n}$. It follows from Lemma 3.3 that $S_{n} \subset L_{h \circ f}, n=1,2, \ldots$ Therefore, $S \subset L_{h \circ f}$ and $f \in C A_{S}^{*}$.

Corollary 3.8. If $f \in C A$ and $S$ is a subset of $[a, b]$ whose intersection with $L_{f}^{c} \sim W_{f}$ is countable, then there exists an $h \in \mathcal{H}$ such that $S \cup W_{f} \cup L_{f} \subset L_{h \circ f}$.

Proof. Combining Theorems 3.4, 3.5, and 3.7 immediately yields the result.

Observe that if $S$ is countable, then Corollary 3.8 implies that $C A_{S}^{*}=C A$. In fact, if $S$ contains no uncountable nullsets, then it is easy to deduce that $C A_{S}^{*}=$ $C A$.

Although it is peripheral to our discussion, we conclude this section by mentioning that Lemma 3.6 has other applications. For example, it can be used to show that if $f$ is any measurable function on $[a, b]$, then there exists a homeomorphism $h$ in $\mathcal{H}$ such that $h \circ f \in L^{p}$ for each $1 \leqslant p$; i.e., $\int_{a}^{b}|h \circ f|^{p}<\infty$ for each $1<p$.

4. Characterizations of $C A^{*}$. In this section we obtain two characterizations of $C A^{*}$ : the first one in Theorem 4.4 and the second in Theorem 4.8. Then, by exploiting these results, we construct approximately continuous functions which cannot be transformed into derivatives, as well as derivatives which are members of $C A \sim C A^{*}$.

Up through the statement of Theorem 4.8 we shall assume that $f$ denotes a function in $C A$ and $P$ denotes the nowhere dense set $\mathrm{Cl}\left(L_{f}^{c}\right)$. We also adopt the following terminology: a nonempty relatively open interval $U$ of a closed set $Q$ is called a portion of $Q$.

We begin with a lemma which not only simplifies our later proofs, but also suggests an approach to the problem of characterizing $C A^{*}$.

THEOREM 4.1. If for every nonempty perfect set $Q \subset \mathrm{Cl}\left(L_{f}^{c}\right)$ there exists a portion $U$ of $Q$ such that $f \in C A_{U}^{*}$, then $f \in C A^{*}$.

Proof. We first show by transfinite induction that for each countable ordinal $\alpha$ $(0 \leqslant \alpha<\Omega)$ there exists a set $Q_{\alpha} \subset P=\mathrm{Cl}\left(L_{f}^{c}\right)$ such that the following three conditions are met:

(i) each $Q_{\alpha}$ is closed,

(ii) $Q_{\alpha} \supset Q_{\gamma}$ whenever $\alpha<\gamma$, and the containment is strict unless $Q_{\alpha}=\varnothing$,

(iii) $f \in C A_{R_{\alpha}}^{*}$, where $R_{\alpha}=[a, b] \sim Q_{\alpha}$.

Write $\mathrm{Cl}\left(L_{f}^{c}\right)=F \cup C$ where $F$ is perfect and $C$ is countable. To begin the induction argment, let $Q_{0}=F$. Then $R_{0} \subset L_{f} \cup C$, which implies by Corollary 3.8 that $f \in C A_{R_{0}}^{*}$. Now suppose that $\beta>0$ is an arbitrary countable ordinal, and that 
for all $0 \leqslant \alpha<\beta$ we have subsets $Q_{\alpha}$ of $P$ satisfying the aforementioned conditions. To complete the induction argument, we show there exists a set $Q_{\beta}$ for which conditions (i)-(iii) are met. If for some $\alpha<\beta$ we have $Q_{\alpha}=\varnothing$, then $f \in C A^{*}$. Therefore, we suppose that $Q_{\alpha} \neq \varnothing$ for all $\alpha<\beta$. We establish the existence of $Q_{\beta}$ according to whether $\beta$ has an immediate predecessor or not:

(1) Suppose $\beta$ has an immediate predecessor, namely $\beta-1$. If $Q_{\beta-1}$ is perfect, then by hypothesis there exists a portion $U$ of $Q_{\beta-1}$ such that $f \in C A_{U}^{*}$. If $Q_{\beta-1}$ is not perfect, there exists a portion of $Q_{\beta-1}$-which we will also denote by $U$-containing precisely one point. By Theorem 3.5, it again follows that $f \in C A_{U}^{*}$. In either case, we define $Q_{\beta}$ to equal $Q_{\beta-1} \sim U$. It is evident that $Q_{\beta}$ is closed and strictly contained in $Q_{\beta-1}$. Moreover, $R_{\beta}=R_{\beta-1} \cup U$. Since $f \in C A_{U}^{*}$ and $f \in$ $C A_{R_{\beta-1}}^{*}$, Theorem 3.7 implies that $f \in C A_{R_{\beta}}^{*}$. Hence, $Q$ satisfies the necessary requirements.

(2) Suppose $\beta$ has no immediate predecessor. In this case, define $Q_{\beta}$ to equal $\cap_{0<\alpha<\beta} Q_{\alpha}$. It is evident from (i) and (ii) that $Q_{\beta}$ is closed, nonempty, and strictly contained in all of its predecessors. By (iii), $f \in C A_{R_{\alpha}}^{*}$ for each of the countably many sets $R_{\alpha}, 0 \leqslant \alpha<\beta$. Since $R_{\beta}=\cup_{0<\alpha<\beta} R_{\alpha}$, it follows from Theorem 3.7 that $f \in C A_{R_{\beta}}^{*}$. Therefore, $Q_{\beta}$ satisfies the specified requirements.

We now conclude by transfinite induction that for each countable ordinal $\alpha$ we have a set $Q_{\alpha}$ satisfying conditions (i)-(iii). By the Cantor-Baire Stationary Principle [13, p. 145], there exists an ordinal $\xi<\Omega$ such that $Q_{\xi}=Q_{\delta}$ for every $\delta>\xi$. By condition (ii) this implies that $Q_{\xi}=\varnothing$. Hence $C A_{R_{\xi}}^{*}=C A^{*}$. Since condition (iii) implies that $f \in C A_{R_{\xi}}^{*}$, the proof is now complete.

Before we can apply Lemma 4.1, we must determine conditions under which there exists some portion $U$ of $Q$ such that $f \in C A_{U}^{*}$. We shall deduce one such necessary and sufficient condition from Lemma 4.3.

For any fixed $x$ in $[a, b]$, approximate continuity guarantees that to each $\alpha>0$ there corresponds a $T_{\alpha}>0$ such that

$$
\lambda\left(I \cap\left\{|f| \geqslant T_{\alpha}\right\}\right) \leqslant \alpha \lambda(I)
$$

whenever $I$ is an interval for which $x \in I \subset[a, b]$. This fact was established in the proof of Theorem 3.5 and was used to construct a homeomorphism $h \in \mathcal{H}$ such that $x \in L_{h \circ f}$. Note that the same $h$ can be constructed even if we only know that for some $\delta>0$ (1) was satisfied whenever $x \in I \subset(x-\delta, x+\delta)$. Nonetheless, the homeomorphism $h$ so obtained depends on $x$, because the numbers $T_{\alpha}$ depend on $x$. However, if $f$ is approximately continuous on some set $S \subset[a, b]$ and we may choose $T_{\alpha}$, for each $\alpha>0$, so that (1) is satisfied independent of $x \in S$, then the proof of Theorem 3.5 shows that we may construct an $h \in \mathcal{H}$ such that $S \subset L_{h \circ f}$. The foregoing discussion prompts us to introduce some new terminology.

Definition 4.2. The function $f$ is called strongly approximately continuous (abbreviated s.a.c.) on $S \subset[a, b]$ if $f$ is approximately continuous on $S$ and if to each $x \in S$ there corresponds a $\delta_{x}>0$, and to each $\alpha>0$ there corresponds a $T_{\alpha}>0$, such that $\lambda\left(I \cap\left\{|f| \geqslant T_{\alpha}\right\}\right) \leqslant \alpha \lambda(I)$ whenever $I$ is an interval for which $x \in I \subset$ $\left(x-\delta_{x}, x+\delta_{x}\right)$. 
It is obvious that $f$ must be bounded on $S$ if $f$ is s.a.c. on $S$. Moreover, by the comments we made preceeding Definition 4.2 , it is evident that $f \in C A_{S}^{*}$ if $f$ is s.a.c. on $S$. This proves half of the following lemma.

LEMMA 4.3. A function $f$ is strongly approximately continuous on $S$ if and only if $f \mid S$ is bounded and $f \in C A_{S}^{*}$.

Proof. It remains only to prove sufficiency, Thus, suppose $f \in C A_{S}^{*}$ and $f \mid S$ is bounded. Then, $f$ is approximately continuous, there is an odd homeomorphism $h$ in $\mathcal{H}$ such that $S \subset L_{h \circ f}$, and there exists $M>0$ such that $|f(x)| \leqslant M$ whenever $x \in S$. It follows that for each $x \in S$,

$$
\varlimsup_{y \rightarrow x} \frac{1}{|y-x|} \int_{x}^{y}|h(f(t))| d t \leqslant h(M) .
$$

If $c=h(M)+1$, then for each $x \in S$ there corresponds a $\delta_{x}>0$ such that

$$
\frac{1}{|y-x|} \int_{x}^{y}|h(f(t))| d t \leqslant c
$$

whenever $0<|y-x|<\delta_{x}$. Therefore, we may deduce that for any $T>0$,

$$
h(T) \lambda((x, y) \cap\{|f| \geqslant T\}) \leqslant c|y-x|
$$

provided that $|y-x|<\delta_{x}$. Now suppose $\alpha>0$ is arbitrary. Since $h$ is increasing without bound, we can find $T_{\alpha}>0$ (independent of $x$ ) such that $h\left(T_{\alpha}\right) \geqslant c / \alpha$. In conjunction with inequality (2), this implies that

$$
\lambda\left((x, y) \cap\left\{|f| \geqslant T_{\alpha}\right\}\right) \leqslant \frac{c}{h\left(T_{\alpha}\right)}|y-x| \leqslant \alpha|y-x|
$$

whenever $|y-x|<\delta_{x}$. It is now evident that $f$ is s.a.c. on $S$, so the proof is complete.

Applying Lemmas 4.3 and 4.1, we now establish our first characterization of $C A^{*}$.

THEOREM 4.4. The function $f$ belongs to $C A^{*}$ if and only if for each nonempty perfect $Q \subset \mathrm{Cl}\left(L_{f}^{c}\right)$ there exists a portion $U$ of $Q$ on which $f$ is strongly approximately continuous.

Proof. To prove necessity, suppose that $f \in C A^{*}$ and that $Q$ is any nonempty perfect set contained in $\mathrm{Cl}\left(L_{f}^{c}\right)$. Since $f$ is Baire 1 , it follows that $f \mid Q$ has a point of continuity. Thus, there exists a portion $U$ of $Q$ on which $f$ is bounded. But $f$ is also in $C A_{U}^{*}$, so Lemma 4.3 implies that $f$ is s.a.c. on $U$.

To prove sufficiency, we now suppose that for each nonempty perfect $Q$ in $\mathrm{Cl}\left(L_{f}^{c}\right)$ there is a portion $U$ on which $f$ is s.a.c. By Lemma $4.3, f \in C A_{U}^{*}$ for each such $U$. It follows immediately from Lemma 4.1 that $f \in C A^{*}$, completing the proof.

Now suppose that $U$ is a portion of some perfect set $Q \subset \mathrm{Cl}\left(L_{f}^{c}\right)$, and that $|f|$ is bounded on $U$ by a number $M$. If $T>M$, then $U$ contributes nothing to the set $\{|f| \geqslant T\}$. Hence, whether or not $f$ is s.a.c. on $U$ (or equivalently, $f \in C A_{U}^{*}$ ) depends solely on the behavior of $f$ on those contiguous intervals to $Q$ whose endpoints intersect $U$. This observation motivates us to consider an alternate characterization of $C A^{*}$. 
To simplify the discussion that follows, we set down a bit more notation. If $Q$ is any nonempty perfect set contained in $P=\operatorname{Cl}\left(L_{f}^{c}\right)$, let $\left\{I_{m}\right\}=\left\{\left(a_{m}, b_{m}\right)\right\}$ denote an enumeration of the intervals contiguous to $Q$. We employ the notation $J \subset * I_{m}$ to mean that $J$ is a subinterval of $I_{m}$ such that $J$ and $I_{m}$ have at least one common endpoint. For each interval $I_{m}$ and $T>0$, we now define a nonnegative number $d_{m}^{T}$ by

$$
d_{m}^{T}=\sup _{J \subset * I_{m}} \frac{\lambda(J \cap\{|f| \geqslant T\})}{\lambda(J)} .
$$

The next lemma exhibits an intimate connection between the numbers $d_{m}^{T}$ and strong approximate continuity.

Lemma 4.5. Let $U$ be a portion of a perfect set $Q \subset \mathrm{Cl}\left(L_{f}^{c}\right)$. Suppose that $f$ is bounded and approximately continuous on $U$ and that for each $\alpha>0$ there exists $a$ $T_{\alpha}>0$ such that $d_{m}^{T_{\alpha}} \leqslant \alpha$ whenever $I_{m}$ has an endpoint in $U$. Then $f$ is strongly approximately continuous on $U$.

Proof. Let $M$ denote a bound for $|f|$ on $U$. Without loss of generality, we shall assume that to each $\alpha>0$ there corresponds a $T_{\alpha}>M$ satisfying the hypothesis of the lemma. Since $U$ is relatively open in $Q$, to each $x \in U$ there corresponds a $\delta_{x}>0$ such that every $I_{m}$ intersecting $\left(x-\delta_{x}, x+\delta_{x}\right)$ has an endpoint in $U$. Thus, if $I_{m} \cap\left(x-\delta_{x}, x+\delta_{x}\right)$ is nonempty, then $d_{m}^{T_{\alpha}} \leqslant \alpha$. Let $I$ be any interval for which $x \in I \subset\left(x-\delta_{x}, x+\delta_{x}\right)$. It is obvious that $I \cap I_{m}$ is either void or is an interval $J$ such that $J \subset * I_{m}$. In either case, we deduce from (3) that $\lambda\left(I \cap I_{m} \cap\left\{|f|>T_{\alpha}\right\}\right)$ $\leqslant \alpha \lambda\left(I \cap I_{m}\right)$. Since $U \cap\left\{|f| \geqslant T_{\alpha}\right\}=\varnothing$, it is evident that

$$
\begin{aligned}
\lambda\left(I \cap\left\{|f| \geqslant T_{\alpha}\right\}\right) & =\sum_{m=1}^{\infty} \lambda\left(I \cap I_{m} \cap\left\{|f| \geqslant T_{\alpha}\right\}\right) \\
& \leqslant \sum_{m=1}^{\infty} \alpha \lambda\left(I \cap I_{m}\right) \leqslant \alpha \lambda(I) .
\end{aligned}
$$

Since $x$ was an arbitrary point in $U$, we conclude that $f$ is s.a.c. on $U$.

As a result of Lemma 4.5, the following definition is useful.

Definition 4.6. Let $U$ be a portion of a perfect set $Q \subset \mathrm{Cl}\left(L_{f}^{c}\right)$. Then $f$ is said to possess property (D) on $U$ if the hypothesis of Lemma 4.5 is satisfied.

Lemma 4.5 may now be rephrased as follows: $f \in C A_{U}^{*}$ if $f$ possesses property (D) on $U$. By writing $\mathrm{Cl}\left(L_{f}^{c}\right)=Q \cup C$ where $Q$ is perfect and $C$ is countable, it is now apparent that $f$ is in $C A^{*}$ if $f$ possesses property (D) on $Q$. This fact, for example, provides another simple proof that the function constructed in Example 3.1 belongs to $C A^{*}$. We note in passing that the converse to Lemma 4.5 is not valid. It is possible for $f$ to be s.a.c. on a portion $U$ of $Q$ even if, for some fixed $\alpha$ and every $T>0, d_{m}^{T}>\alpha$ for infinitely many $I_{m}$ which have an endpoint in $U$.

By combining Lemma 4.5 and Theorem 4.4, we immediately obtain another sufficient condition for $f$ to be in $C A^{*}$. We shall demonstrate in Theorem 4.8 that this condition is also necessary; however, we first establish another lemma. We assume that $\left\{I_{m}\right\}$ denotes the intervals contiguous to any nonempty nowhere dense perfect subset $Q$ of $[a, b]$. 
LEMMA 4.7. Let $\left\{L_{j}\right\}=\left\{\left(c_{j}, d_{j}\right)\right\}$ be a subsequence of $\left\{I_{m}\right\}$ such that the set $E=\left\{t: t\right.$ is an endpoint of some $\left.L_{j}\right\}$ is dense in some portion $U$ of $Q$. If $z_{j} \in\left(c_{j}, d_{j}\right)$ for each $j$, then the following are true:

(a) for some $x \in U$ there exists a subsequence $\left\{z_{j_{n}}\right\}$ of $\left\{z_{j}\right\}$ such that $z_{j_{n}}$ approaches $x$ monotonically from the right and

$$
\varlimsup_{n \rightarrow \infty} \frac{\lambda\left(\left(x, z_{j_{n}}\right) \cap \cup_{j=1}^{\infty}\left(c_{j}, z_{j}\right)\right)}{z_{j_{n}}-x} \geqslant \frac{1}{2},
$$

(b) for some $x \in U$ there exists a subsequence $\left\{z_{j_{n}}\right\}$ such that $z_{j_{n}}$ approaches $x$ monotonically from the left and

$$
\varlimsup_{n \rightarrow \infty} \frac{\lambda\left(\left(z_{j_{n}}, x\right) \cap \cup_{j=1}^{\infty}\left(z_{j}, d_{j}\right)\right)}{x-z_{j_{n}}} \geqslant \frac{1}{2} .
$$

Proof. For convenience we assume that $U=Q$. By induction on $n$, we construct a decreasing subsequence $\left\{L_{j_{n}}\right\}=\left\{\left(c_{j_{n}}, d_{j_{n}}\right)\right\}$ of $\left\{L_{j}\right\}$ and an increasing sequence $\left\{x_{n}\right\}$ from $E$, such that $0<c_{j_{n}}-x_{n}<z_{j_{n}}-c_{j_{n}}$. For $n=1$, choose $L_{j_{1}}$ to be the first term in $\left\{L_{j}\right\}$ not containing $a$, and pick $x_{1}$ in $E$ so that $0<c_{j_{1}}-x_{1}<$ $z_{j_{1}}-c_{j_{1}}$. This is possible since $E$ is dense in $Q$. Now assume that for each $n$ less than $k$ we have chosen $L_{j_{n}}$ and $x_{n}$ with the desired properties. Since $E$ is dense in $Q$, we may choose from $\left\{L_{j}\right\}$ an interval $L_{j_{k}}$ such that $L_{j_{k}} \subset\left(x_{k-1}, c_{j_{k-1}}\right)$ and $j_{k}>j_{k-1}$. We may then pick a point $x_{k}$ from $E$ such that $x_{k}>x_{k-1}$ and $0<c_{j_{k}}-x_{k}<z_{j_{k}}-$ $c_{j_{k}}$. This completes the induction step.

Since $Q$ is closed and bounded, $\left\{x_{n}\right\}$ converges to a point $x$ in $Q$. It is evident from our construction that the sequence $\left\{z_{j_{n}}\right\}$ converges monotonically to $x$ from the right. Furthermore, for each $n$,

$$
\frac{z_{j_{n}}-c_{j_{n}}}{z_{j_{n}}-x}>\frac{z_{j_{n}}-c_{j_{n}}}{z_{j_{n}}-x_{n}}>\frac{z_{j_{n}}-c_{j_{n}}}{2\left(z_{j_{n}}-c_{j_{n}}\right)}=\frac{1}{2} .
$$

Since

$$
\frac{\lambda\left(\left(x, z_{j_{n}}\right) \cap \cup_{j=1}^{\infty}\left(c_{j}, z_{j}\right)\right)}{z_{j_{n}}-x}>\frac{z_{j_{n}}-c_{j_{n}}}{z_{j_{n}}-x},
$$

it follows that (4) is valid. This verifies (a); the validity of (b) can be established by a symmetric argument.

THEOREM 4.8. The function $f$ belongs to $C A^{*}$ if and only if for each nonempty perfect $Q \subset \mathrm{Cl}\left(L_{f}^{c}\right)$ there is a portion $U$ of $Q$ on which $f$ possesses property (D).

Proof. Sufficiency has already been verified; to verify necessity we shall establish the contrapositive statement. Suppose there exists a nonempty perfect $Q \subset P$ such that $f$ does not possess property (D) on any portion of $Q$. We shall show that this implies $|h \circ f| \notin \Lambda$ for any odd $h$ in $\mathcal{H}$. Since $f$ is Baire $1, f \mid Q$ has a point of continuity. Hence, there exists a portion $V$ of $Q$ such that $f \mid V$ is bounded but $f$ does not possess property (D) on any portion of $Q$ contained in $V$. Purely for convenience, we shall suppose that $V=Q$. This assumption implies that $f \mid Q$ is bounded by a number $M_{1}>0$, and for every portion $U$ of $Q$ there is an $\alpha>0$ such that, for each $T>0, d_{m}^{T}>\alpha$ for some $I_{m}$ with an endpoint in $U$. 
Let $h$ be any odd homeomorphism in $\mathscr{H}$ and let $M$ denote the positive number $h\left(M_{1}\right)$. Since $h$ is increasing without bound, we observe that, for each $\alpha>0$, $h(T)>4 M / \alpha$ for some sufficiently large $T>0$. Let $\left\{U_{j}\right\}$ be a countable collection of portions of $Q$ which forms a base for $Q$. We deduce that for each $j$ there exist positive numbers $\alpha_{j}$ and $T_{j}$, and an interval $I_{m}$, such that the following three conditions are met:

(i) $d_{m_{j}}^{T_{j}}>2 \alpha_{j}$,

(ii) $h\left(T_{j}\right)>4 M / \alpha_{j}$,

(iii) $I_{m}$ has an endpoint in $U_{j}$.

Denote the resulting subsequence $\left\{I_{m}\right\}$ of $\left\{I_{m}\right\}$ simply by $\left\{L_{j}\right\}=\left\{\left(c_{j}, d_{j}\right)\right\}$. By (3) and (i) above, we conclude that for each $j$ at least one of the following two statements must be true:

(*) there exists a $z_{j} \in\left(c_{j}, d_{j}\right)$ such that

$$
\frac{\lambda\left(\left(c_{j}, z_{j}\right) \cap\left\{|f| \geqslant T_{j}\right\}\right)}{z_{j}-c_{j}}>\alpha_{j}
$$

or

(**) there exists a $z_{j} \in\left(c_{j}, d_{j}\right)$ such that

$$
\frac{\lambda\left(\left(z_{j}, d_{j}\right) \cap\left\{|f| \geqslant T_{j}\right\}\right)}{d_{j}-z_{j}}>\alpha_{j} .
$$

Let $E_{1}$ denote the set of endpoints of those $I_{j}$ for which (*) is valid, and $E_{2}$ denote the like set for which (**) is valid. From (iii) it follows that $E_{1} \cup E_{2}$ is dense in $Q$. Hence, $E_{1}$ or $E_{2}$ must be dense in some portion $U$ of $Q$. We only consider the case where $E_{1}$ is dense in $U$-the other case can be treated similarly. Let the subsequence of $\left\{L_{j}\right\}$ consisting of those intervals having an endpoint in $E_{1}$ also be denoted by $\left\{L_{j}\right\}$. Thus, we are assuming that (*), (i), (ii), and (iii) are valid for every $j$. By Lemma 4.7, for some $x \in U$ there exists a subsequence $\left\{z_{j_{n}}\right\}$ of $\left\{z_{j}\right\}$ such that $z_{j_{n}}$ approaches $x$ monotonically from the right and (4) is valid. Observe that by (ii) and $(*)$,

$$
\begin{aligned}
\frac{1}{z_{j}-c_{j}} \int_{c_{j}}^{z_{j}}|h(f(t))| d t & =\frac{1}{z_{j}-c_{j}} \int_{c_{j}}^{z_{j}} h(|f(t)|) d t \\
& \geqslant \frac{1}{z_{j}-c_{j}} h\left(T_{j}\right) \lambda\left(\left(c_{j}, z_{j}\right) \cap\left\{|f|>T_{j}\right\}\right)>\frac{4 M}{\alpha_{j}} \cdot \alpha_{j}=4 M,
\end{aligned}
$$

for each $j$. Since $\left(x, z_{j_{n}}\right) \cap\left(c_{j}, z_{j}\right)$ is either empty or equals $\left(c_{j}, z_{j}\right)$, we deduce from (4) and (5) that

$$
\begin{aligned}
\varlimsup_{n \rightarrow \infty} \frac{1}{z_{j_{n}}-x} \int_{x}^{z_{j_{n}}}|h(f(t))| d t & >\varlimsup_{n \rightarrow \infty} \frac{1}{z_{j_{n}}-x} \sum_{j=1}^{\infty} \int_{\left(x, z_{j}\right) \cap\left(c_{j}, z_{j}\right)}|h(f(t))| d t \\
& >\varlimsup_{n \rightarrow \infty} \frac{1}{z_{j_{n}}-x} \sum_{j=1}^{\infty} 4 M \lambda\left(\left(x, z_{j_{n}}\right) \cap\left(c_{j}, z_{j}\right)\right) \\
& =\varlimsup_{n \rightarrow \infty} 4 M \frac{\lambda\left(\left(x, z_{j_{n}}\right) \cap \cup_{j=1}^{\infty}\left(c_{j}, z_{j}\right)\right)}{z_{j_{n}}-x}>2 M .
\end{aligned}
$$


But $|h(f(x))| \leqslant M$, since $x \in Q$. Therefore, $|h \circ f|$ is not in $\Lambda$. Since $h$ was an arbitrary odd homeomorphism in $\mathcal{H}$, this implies that $f \notin C A^{*}$. The proof is now complete.

Although we have consistently intimated that the containment of $C A^{*}$ in $C A$ is proper, we have yet to verify this fact formally. By capitalizing on Theorem 4.8 , we shall demonstrate in Theorem 4.10 below that $C A \sim C A^{*}$ is indeed nonvoid. First, we consider a lemma which will play a fundamental role in our construction of functions in $C A \sim C A^{*}$.

LEMMA 4.9. Suppose $P$ is any nonempty nowhere dense perfect subset of $[a, b]$ and $\Upsilon$ is the collection of intervals contiguous to $P$. Then $\Upsilon$ can be decomposed into denumerably many disjoint sequences of intervals

$$
\left\{J_{k}^{(n)}\right\}, \quad \begin{aligned}
& n=1,2, \ldots, \\
& k=1,2, \ldots,
\end{aligned}
$$

such that, if $B_{n}$ denotes the right endpoint of $J_{1}^{(n)}$, the following conditions are met:

(i) for $k>1, J_{k}^{(n)}$ approaches $B_{n}$ monotonically from the right and is contained in $\left(B_{n}, B_{n}+1 / n\right)$,

(ii) $d\left(\cup_{k=2}^{\infty} J_{k}^{(n)}, B_{n}\right)=0$,

(iii) $\left\{B_{n}: n=1,2, \ldots\right\}$ is a dense subset of $P$.

Proof. First decompose the collection $\Upsilon$ into a denumerable number of disjoint subcollections $\Upsilon_{n}, n=1,2, \ldots$, with the property that the endpoints of the intervals in $\Upsilon_{n}$ form a dense subset of $P$. (For brevity we shall say simply that $\Upsilon_{n}$ is dense in $P$.) This decomposition is possible because the collection $\Upsilon$ is order isomorphic to the rationals, and the rationals can be decomposed into a denumerable number of disjoint dense subsets.

Let $\left\{I_{m}\right\}$ be an enumeration of the intervals in $\Upsilon$. By induction on $n$, we now proceed to construct disjoint sequences $\left\{J_{k}^{(n)}\right\}$ such that $J_{k}^{(n)} \in \Upsilon_{n}$ for $k>1$ and conditions (i) and (ii) above are met. For $n=1$ we let $J_{1}^{(1)}=I_{1}$. Since $\Upsilon_{1}$ is dense in $P$, it is clear that we may choose intervals $J_{k}^{(1)}, k=2,3, \ldots$, from $\Upsilon_{1}$ such that the sequence $\left\{J_{k}^{(1)}\right\}$ meets conditions (i) and (ii). Now assume that for each $n$ less than $r$ we have obtained disjoint sequences $\left\{J_{k}^{(n)}\right\}$ such that $J_{k}^{(n)} \in \Upsilon_{n}$ for $k>1$ and (i) and (ii) are valid. Choose $J_{1}^{(r)}$ to be the first interval out of the enumeration $\left\{I_{m}\right\}$ which is different from $J_{k}^{(n)}, n=1,2, \ldots, r-1, k=1,2, \ldots$ Such an interval is guaranteed to exist because all but at most $(r-1)$ of the infinitely many intervals in $\Upsilon_{r}$ are still remaining. Since $\Upsilon_{r}$ is dense in $P$, it is again clear that we may choose intervals $J_{k}^{(r)}, k=2,3, \ldots$, from $\Upsilon_{r}$ such that (i) and (ii) are satisfied and $J_{k}^{(r)} \neq J_{1}^{(n)}$ for any $n<r$. This establishes the induction step. Therefore, for each $n$ we have a sequence $\left\{J_{k}^{(n)}\right\}$ such that $J_{k}^{(n)} \in \Upsilon_{n}$ for $k>1$ and (i) and (ii) are satisfied. It is also apparent by our construction that these sequences are disjoint and that each $I_{m}$ in $\Upsilon$ is equal to exactly one $J_{k}^{(n)}$.

To finish the proof, we now show that condition (iii) is also satisfied for the sequences constructed above. Suppose there is a portion $U$ of $P$ which does not contain a right endpoint of $J_{1}^{(n)}$ for any $n$. Then from (i) it follows that there must exists a large enough integer $N$ and a portion $U_{1}$ of $P$ such that $U_{1} \subset U$ and $U_{1}$ 
does not contain an endpoint of $J_{k}^{(N)}$ for any $k$. However, $\Upsilon_{N}$ is dense in $P$, so $U_{1}$ contains a right endpoint of some interval $I_{M}$ from $\Upsilon_{N}$. Since the collections $\Upsilon_{n}$ are disjoint and $J_{k}^{(n)} \in \Upsilon_{n}, k=2,3, \ldots, n=1,2, \ldots$, we deduce that $I_{M}$ equals $J_{1}^{(n)}$ for some $n$. We have now reached a contradiction. Thus (iii) is satisfied and our proof is complete.

To facilitate upcoming constructions, we introduce some descriptive terminology. Suppose that $I=[\alpha, \beta]$ is an interval and that $J$ is the interval with midpoint $(\alpha+\beta) / 2$ and length $\lambda(I) / 2$. Then a function $f$ is called trapezoidal of height $s$ on $I$ if $f(\alpha)=f(\beta)=0, f(t)=s$ for all $t \in J$, and $f$ is defined elsewhere on $I$ by linear extension. It is evident that $\int_{l} f=\frac{3}{4} s \lambda(I)$ for such an $f$.

THEOREM 4.10. Let $P$ be a nonempty nowhere dense perfect nullset in $[a, b]$. Then there exists a nonnegative summable function $f \in C A \sim C A^{*}$ such that $L_{f}^{c}=P$ and $L_{h \circ f}^{c}$ is dense in $P$ for any $h \in \mathcal{H}$.

Proof. Let $\left\{I_{m}\right\}=\left\{\left(a, b_{m}\right)\right\}$ be an enumeration of the intervals contiguous to $P$. Decompose the collection of these intervals into denumerably many disjoint sequences

$$
\left\{J_{k}^{(n)}\right\}, \quad \begin{aligned}
& n=1,2, \ldots, \\
& k=1,2, \ldots,
\end{aligned}
$$

such that the conditions specified in Lemma 4.9 are satisfied. Since each $I_{m}$ is a term of precisely one of these sequences, we may define a function $\Phi$, from the natural numbers onto itself, by setting $\Phi(m)=n$ if and only if $I_{m}=J_{k}^{(n)}$ for some $k$. Let $\left\{s_{m}\right\}$ be an unbounded increasing sequence of positive numbers such that $\sum_{m=1}^{\infty} s_{m} \lambda\left(I_{m}\right)<\infty$. (It is easy to verify that such a sequence must exist.) For each $m$, define $\left[\alpha_{m}, \beta_{m}\right]$ to be the closed subinterval of $I_{m}$ having midpoint $\left(a_{m}+b_{m}\right) / 2$ and length $\lambda\left(I_{m}\right) /(\Phi(m)+1)$. Now, let $f$ be the nonnegative function defined as follows: $f$ is trapezoidal of height $4(\Phi(m)+1) s_{m} / 3$ on $\left[\alpha_{m}, \beta_{m}\right], m=1,2, \ldots$, while $f$ is identically 0 on $[a, b] \sim\left(\cup_{m=1}^{\infty}\left[\alpha_{m}, \beta_{m}\right]\right)$. We shall verify that $f$ possesses all the required properties.

Observe that for each $m$,

$$
\int_{I_{m}} f=\int_{\alpha_{m}}^{\beta_{m}} f=\frac{3}{4}\left(\frac{4(\Phi(m)+1)}{3} s_{m}\right) \frac{\lambda\left(I_{m}\right)}{\Phi(m)+1}=s_{m} \lambda\left(I_{m}\right) .
$$

Since $s_{m} \rightarrow \infty$ and $P$ is a nullset, it is easy to deduce that

$$
\varlimsup_{y \rightarrow x} \frac{1}{|y-x|} \int_{x}^{y} f(t) d t=\infty
$$

for each $x \in P$. This implies that $P \subset L_{f}^{c}$. Moreover, $f$ is continuous on $P^{c}$, so we conclude that $L_{f}^{c}=P$. Note also that $\int_{a}^{b} f=\sum_{m=1}^{\infty} s_{m} \lambda\left(I_{m}\right)<\infty$, so $f$ is summable.

Let $E=\{f>0\}$. To verify that $f \in C A$ it clearly suffices to demonstrate that $d(E, x)=0$ for any $x$ in $P$. To simplify this task, associate with each $I_{m}$ a number $e_{m}$ defined by

$$
e_{m}=\sup _{J \subset * I_{m}} \frac{\lambda(J \cap E)}{\lambda(J)}
$$


Here, once again, $J \subset * I_{m}$ means that $J$ is a subinterval of $I_{m}$ such that $J$ and $I_{m}$ have at least one common endpoint. It is easy to see that

$$
e_{m}<\frac{\lambda\left(I_{m}\right)}{\Phi(m)+1} \div \frac{\lambda\left(I_{m}\right)}{2}=\frac{2}{\Phi(m)+1} .
$$

Let $x$ be an arbitrary point in $P$. Then if $y \neq x,(x, y) \cap I_{m}$ is either empty or is an interval $J$ such that $J \subset * I_{m}$. Therefore, we may deduce from (6) and (7) that

$$
\lambda\left(E \cap(x, y) \cap I_{m}\right)<\frac{2}{\Phi(m)+1} \lambda\left((x, y) \cap I_{m}\right) .
$$

We now proceed to verify that $d^{+}(E, x)=0$. Suppose that $x$ is not a left endpoint of some $I_{m}$, or there is nothing to prove. There are two cases to consider:

(i) Suppose that $x$ is not a right endpoint of $J_{1}^{(n)}$ for any $n$. Let $\varepsilon>0$. Choose an integer $q$ so that $2 /(n+1)<\varepsilon$ whenever $n \geqslant q$. Due to the properties that the sequences $\left\{J_{k}^{(n)}\right\}$ possess, it is clear that we may choose $\delta>0$ such that $0<y-x$ $<\delta$ implies that $\Phi(M) \geqslant q$ whenever $I_{m} \cap(x, y)$ is nonempty. It now follows from (8) that

$$
\lambda\left(E \cap(x, y) \cap I_{m}\right)<\varepsilon \lambda\left((x, y) \cap I_{m}\right), \quad m=1,2, \ldots,
$$

whenever $0<y-x<\delta$. Hence,

$$
\lambda(E \cap(x, y))=\sum_{m=1}^{\infty} \lambda\left(E \cap(x, y) \cap I_{m}\right)<\sum_{m=1}^{\infty} \varepsilon \lambda\left((x, y) \cap I_{m}\right)=\varepsilon(y-x),
$$

whenever $0<y-x<\delta$. This implies that $d^{+}(E, x)=0$.

(ii) Suppose that $x$ is right endpoint of $J_{1}^{(N)}$. Let $\varepsilon>0$. Choose $q$ to be an integer so that $2 /(n+1)<\varepsilon / 2$ whenever $n \geqslant q$. It is evident we may choose $\delta>0$ such that $0<y-x<\delta$ implies that $\Phi(m)=N$ or $\Phi(m) \geqslant q$ whenever $I_{m} \cap(x, y)$ is nonempty. Since $d\left(\cup_{k=2}^{\infty} J_{k}^{(N)}, x\right)=0$, we may assume that $\delta$ was also chosen so small that $0<y-x<\delta$ implies that

$$
\lambda\left(\bigcup_{k=2}^{\infty} J_{k}^{(N)} \cap(x, y)\right)<\frac{\varepsilon}{2}(y-x) .
$$

Hence, $0<y-x<\delta$ implies that

$$
\begin{aligned}
\lambda(E \cap(x, y)) & =\sum_{m=1}^{\infty} \lambda\left(E \cap(x, y) \cap I_{m}\right) \\
& =\sum_{\Phi(m)=N} \lambda\left(E \cap(x, y) \cap I_{m}\right)+\sum_{\Phi(m)>q} \lambda\left(E \cap(x, y) \cap I_{m}\right) \\
& <\frac{\varepsilon}{2}(y-x)+\frac{\varepsilon}{2}(y-x)=\varepsilon(y-x),
\end{aligned}
$$

where the second $\varepsilon(y-x) / 2$ is obtained as in (i). This shows that $d^{+}(E, x)=0$.

A similar argument to (i) above can be used to show that $d^{-}(E, x)=0$. Therefore, $d(E, x)=0$ for each $x$ in $P$, so $f$ belongs to $C A$.

To complete the proof it suffices to show that $L_{h \circ f}^{c}$ is dense in $P$ for any $h \in \mathcal{H}$. For each interval $I_{m}$ and each $T>0$, recall that

$$
d_{m}^{T}=\sup _{J \subset * I_{m}} \frac{\lambda(J \cap\{|f| \geqslant T\})}{\lambda(J)} .
$$


Based on how $f$ is defined, simple estimates show that if

$$
0<T \leqslant 4(\Phi(m)+1) s_{m} / 3
$$

then

$$
d_{m}^{T}>\frac{\frac{1}{2}\left(\beta_{m}-\alpha_{m}\right)}{\lambda\left(I_{m}\right)}=\frac{1}{2(\Phi(m)+1)} .
$$

In particular, if we denote $4(\Phi(m)+1) s_{m} / 3$ simply by $T_{m}$, then

$$
d_{m}^{T_{m}} \geqslant \frac{1}{2(\Phi(m)+1)}, \quad m=1,2, \ldots
$$

Now let $U$ be an arbitrary portion of $P$, and let $\Upsilon_{U}$ be the collection of all those contiguous intervals to $P$ which have an endpoint in $U$. Since the right endpoints of the intervals $J_{1}^{(n)}, n=1,2, \ldots$, form a dense subset of $P, U$ contains a right endpoint of $J_{1}^{(N)}$ for some $N$. But this imples that $J_{k}^{(N)} \in \Upsilon_{U}$ for infinitely many $k$. Thus, we deduce that

$$
\left\{T_{m}: \Phi(m)=N \text { and } I_{m} \in \Upsilon_{U}\right\}
$$

is an infinite set. Set $\alpha=1 / 2(N+1)$ and let $T$ be any positive number. The set appearing in (10) is unbounded (because $s_{m} \rightarrow \infty$ ), so there exits an integer $M$ such that $T_{M}>T, \Phi(M)=N$, and $I_{M} \in \Upsilon_{U}$. It then follows from (9) that $d_{M}^{T}>d_{M}^{T_{M}}>$ $1 / 2(N+1)=\alpha$. This implies that $f$ does not possess property (D) on $U$. From Theorem 4.8 we conclude that $f \notin C A^{*}$. Furthermore, we have shown that $f$ does not possess property (D) on any portion of $P$; therefore, it is clear that the argument given in the proof of Theorem 4.8 may be used to demonstrate that $f \notin C A_{V}^{*}$ for any portion $V$ of $P$. This implies that $L_{h \circ f}^{c}$ is dense in $P$, so the proof is complete.

If $S$ is any uncountable Borel subset of $[a, b]$, then $S$ contains a nonempty nowhere dense perfect nullset. Thus, for any such $S$, Theorem 4.10 shows that $C A_{S}^{*}$ is properly contained in $C A$. Note that for any $f \in C A \sim C A_{S}^{*}$ and $h \in \mathcal{K}$, Corollary 3.8 implies that the set of firm non-Lebesgue points of $h \circ f$ must be uncountable.

The nonnegative function constructed in the proof of Theorem 4.10 cannot be transformed into a derivative via outer composition with any homeomorphism in $\mathcal{H}$. However, the next theorem demonstrates that there are summable derivatives belonging to $C A \sim C A^{*}$-such functions are derivatives of their integrals due to "cancellation."

THEOREM 4.11. Let $P$ be a nonempty nowhere dense pefect nullset in $[a, b]$. Then there exists a summable approximately continuous derivative $f$ such that $L_{h}^{c}$ 。f is dense in $P$ for any $h \in \mathcal{H}$.

Proof. Let $\left\{I_{m}\right\},\left\{J_{k}^{(n)}\right\}, \Phi,\left\{s_{m}\right\}$, and $\left\{\left[\alpha_{m}, \beta_{m}\right]\right\}$ have exactly the same meaning as in the proof of Theorem 4.10. Define $f$ to be identically 0 on $[a, b] \sim$ $\left(\cup_{m=1}^{\infty}\left[\alpha_{m}, \beta_{m}\right]\right)$. Next, partition each $\left[\alpha_{m}, \beta_{m}\right]$ into $2 s_{m}(\Phi(m)+1)$ subintervals of equal length. If for fixed $m$ these subintervals are enumerated preceding from left 
to right, then on each subinterval of odd index define $f$ to be trapezoidal of height $\frac{4}{3}(\Phi(m)+1) s_{m}$, while on each subinterval of even index define $f$ to be trapezoidal of height $\left(-\frac{4}{3}\right)(\Phi(m)+1) s_{m}$.

For each $m$, observe that $\int_{I_{m}} f=0$ and $\int_{I_{m}}|f|=\int_{\alpha_{m}}^{\beta_{m}}|f|=s_{m} \lambda\left(I_{m}\right)$. By slightly modifying the arguments provided in the proof of Theorem 4.10 , it is easy to demonstrate that $f$ is a summable function in $C A \sim C A^{*}$ and that, for every $h \in \mathcal{H}, L_{h \circ f}^{c}$ is dense in $P=L_{f}^{c}$.

To complete the proof we establish that $f$ is the derivative of its integral. Since $f$ is continuous on $P^{c}$ and $f \mid P$ is identically 0 , it suffices to verify that

$$
\lim _{y \rightarrow x} \frac{1}{y-x} \int_{x}^{y} f(t) d t=0
$$

for each $x \in P$. We shall only demonstrate that the right-sided limit is 0 , because a symmetric argument can be applied to treat the left-sided limit. The result is obvious if $x$ is a left endpoint of some $I_{m}$, so assume that $x$ is any other type of point in $P$. Note that the definition of $f$ implies that

$$
\begin{aligned}
\left|\frac{1}{z-a_{m}} \int_{a_{m}}^{z} f(t) d t\right| & \leqslant \frac{1}{\alpha_{m}-a_{m}}\left|\int_{a_{m}}^{z} f(t) d t\right| \\
& \leqslant \frac{4}{\lambda\left(I_{m}\right)} \cdot \frac{3}{4} \cdot \frac{4}{3}(\Phi(m)+1) s_{m} \cdot \frac{\lambda I_{m}}{2(\Phi(m)+1)^{2} s_{m}} \\
& =\frac{2}{\Phi(m)+1},
\end{aligned}
$$

whenever $z \in I_{m}=\left(a_{m}, b_{m}\right)$. Let $\varepsilon>0$. We shall show that there exists $\delta>0$ such that

$$
\left|\frac{1}{y-x} \int_{x}^{y} f(t) d t\right|<\varepsilon
$$

whenever $0<y-x<\delta$. There are two cases to consider:

(i) Suppose $x$ is not an endpoint of $J_{1}^{(n)}$ for any $n$. Choose $q$ such that $2 /(n+1)<\varepsilon$ for all $n \geqslant q$. As in the proof of Theorem 4.10, we may find a $\delta>0$ for which $0<y-x<\delta$ implies that $\Phi(m) \geqslant q$ whenever $I_{m} \cap(x, y)$ is nonempty. Therefore, from (11) we deduce that

$$
\begin{aligned}
\left|\frac{1}{y-x} \int_{x}^{y} f(t) d t\right| & \leqslant \frac{1}{y-x} \sum_{m=1}^{\infty}\left|\int_{I_{m} \cap(x, y)} f(t) d t\right| \\
& <\frac{1}{y-x} \sum_{m=1}^{\infty} \varepsilon \lambda\left(I_{m} \cap(x, y)\right)=\varepsilon,
\end{aligned}
$$

whenever $0<y-x<\delta$.

(ii) Suppose $x$ is a right endpoint of $J_{1}^{(N)}$ for some $N$. Pick $q$ so that $2 /(n+1)<$ $\varepsilon / 2$ whenever $n \geqslant q$. By the same reasoning that was used in the proof of Theorem 4.10, we may find a $\delta>0$ such that $0<y-x<\delta$ implies that

$$
\lambda\left(\bigcup_{k=2}^{\infty} J_{k}^{(N)} \cap(x, y)\right)<\frac{\varepsilon}{2}(y-x)
$$


and $\Phi(m)=N$ or $\Phi(m) \geqslant q$ whenever $I_{m} \cap(x, y)$ is nonempty. Therefore, it is evident that whenever $0<y-x<\delta$,

$$
\begin{aligned}
\left|\frac{1}{y-x} \int_{x}^{y} f(t) d t\right| & <\frac{\varepsilon}{2}+\frac{1}{y-x} \sum_{k=2}^{\infty}\left|\int_{(x, y) \cap J_{k}^{(N)}} f(t) d t\right| \\
& <\frac{\varepsilon}{2}+\frac{1}{y-x} \sum_{k=2}^{\infty} \frac{2}{N+1} \lambda\left(J_{k}^{(N)} \cap(x, y)\right)<\frac{\varepsilon}{2}+\frac{2}{N+1} \cdot \frac{\varepsilon}{2} \leqslant \varepsilon
\end{aligned}
$$

The proof is now complete.

Let $C A^{* *}=\{f \in C A: h \circ f \in \Delta$ for some $h \in \mathcal{C}\}$. It is evident that $C A^{*} \subset$ $C A^{* *} \subset C A$, and the last two theorems have shown these containments are strict. Although we have provided characterizations of the class $C A^{*}$, we are unaware of a characterization of the class $C A^{* *}$.

To provide added perspective to $C A^{*}$, we conclude this section with a remark concerning the connection between $C A^{*}$ and $\Delta_{2}=\left\{g: g \in \Delta\right.$ and $\left.g^{2} \in \Delta\right\}$. It is not difficult to demonstrate that a function $f$ belongs to $C A^{*}$ if and only if there exists an $h \in \mathcal{H}$ such that every $x \in[a, b]$ is a second order Lebesgue point of $h \circ f$ (i.e., $\left.\lim _{y \rightarrow x}[1 /(y-x)] \int_{x}^{y}|h(f(t))-h(f(x))|^{2} d t=0\right)$. Iosifescu proved in [9] that every point of $[a, b]$ is a second order Lebesgue point of a function $g$ if and only if $g \in \Delta_{2}$. Thus, we deduce that $C A^{*}$ consists of those functions which can be homeomorphically transformed into functions which together with their squares are derivatives; i.e., $\mathcal{H} \circ \Delta_{2}=C A^{*}$.

5. The smallest algebra generated from $\Lambda$. In this final section we show that, unlike $\Lambda, C A^{*}$ is an algebra of functions. This motivates us to consider the smallest algebra of functions generated from $\Lambda$, which we denote by $\Lambda_{*}$. In Theorem 5.3 we obtain a characterization of $\Lambda_{*}$ which, when contrasted with our characterizations of $C A^{*}$, is very simple. Then, in Theorem 5.4, we establish that every function in $\Lambda_{*}$ may be transformed into $\Lambda$ by a single suitable homeomorphic change of scale.

The proof that $C A^{*}$ is an algebra rests primarily on the following easy lemma.

Lemma 5.1. (a) Suppose $k \geqslant 0, f \in C A^{*}, g \in C A$, and $|g(t)| \leqslant|f(t)|$ whenever $t \in\{|g| \geqslant k\}$. Then $g \in C A^{*}$.

(b) $C A^{*}$ is closed under outside composition with continuous functions from $R$ into $R$.

(c) If $f \in C A^{*}$ and $g \in C A^{*}$, then $\max (f, g) \in C A^{*}$.

Proof. (a) There exists an odd $h \in \mathcal{H}$ such that $h \circ f \in \Lambda$. Let $x \in[a, b]$. Choose

$$
c=\max (|h(f(x))|+1, h(k)),
$$

and let $B=\{|h \circ g|>|h(g(x))|+c\}$ and $B_{1}=\{|h \circ f|>|h(f(x))|+1\}$. Since $|h(g(t))| \leqslant|h(f(t))|$ whenever $|h(g(t))| \geqslant h(k)$, it follows that $B \subset B_{1}$ and

$$
\frac{1}{|y-x|} \int_{B}|h(g(t))| d t \leqslant \frac{1}{|y-x|} \int_{B_{1}}|h(f(t))| d t .
$$

Applying Lemma 2.2 to $h \circ f$ and then to $h \circ g$, we deduce that $x \in L_{h \circ g}$. 
(b) Let $G$ be a continuous function on $R$ and let $f \in C A^{*}$. Clearly, we may find a homeomorphism $h \in \mathcal{H}$ such that $|G(u)| \leqslant|h(u)|$ for all $|u| \geqslant 1$. Let $k$ be an upper bound for $|G|$ on $[-1,1]$. Then $|G(f(t))| \leqslant|h(f(t))|$ whenever $t \in\{|G \circ f|>$ $k$ \}. Since $h \circ f \in C A^{*}$ and $G \circ f \in C A$, part (a) implies that $G \circ f \in C A^{*}$.

(c) If $f \in C A^{*}$ and $g \in C A^{*}$, then there exists $h \in \mathcal{H}$ such that $h \circ f \in \Lambda$ and $h \circ g \in \Lambda$. Since $\max (h \circ f, h \circ g) \in \Lambda$ and $\max (h \circ f, h \circ g)=h \circ(\max (f, g))$, it follows that $\max (f, g) \in C A^{*}$.

THEOREM 5.2. $C A^{*}$ is a uniformly closed algebra of functions.

Proof. $C A^{*}$ is obviously closed under scalar multiplication. Let $f \in C A^{*}$ and $g \in C A^{*}$. Since $|f+g| \leqslant 2 \max (|f|,|g|)$, we deduce from Lemma 5.1 that $f+g$ is in $C A^{*}$. The squaring function is continuous, so Lemma 5.1 implies that the square of any function belonging to $C A^{*}$ also belongs to $C A^{*}$. Hence,

$$
\left[(f+g)^{2}-f^{2}-g^{2}\right] / 2 \in C A^{*} \text {. }
$$

This shows that $f g \in C A^{*}$.

It remains only to verify that $C A^{*}$ is closed under uniform convergence. Suppose $d>0, f \in C A^{*}, g \in C A$, and $|g(t)|<|f(t)|+d$ for each $t$. Then $g \in C A^{*}$, by Lemma 5.1. Since the uniform limit of approximately continuous functions is approximately continuous, it is now clear that $C A^{*}$ is a uniformly closed algebra.

The algebra $\Lambda_{*}$ consists of functions which arise as sums of products of members of $\Lambda$. However, note that in the course of proving our next theorem, we establish that every function in $\Lambda_{*}$ is expressible as a product of functions in $\Lambda$.

THEOREM 5.3. A function $f$ belongs to $\Lambda_{*}$ if and only if it is approximately continuous and there exists some positive integer $N$ such that

$$
\varlimsup_{y \rightarrow x} \frac{1}{|y-x|} \int_{x}^{y} \sqrt[N]{|f(t)|} d t<\infty
$$

for each $x \in[a, b]$.

Proof. To prove necessity, suppose first that $f \in \Lambda_{*}$. This implies the existence of functions $F_{1}, F_{2}, \ldots, F_{m}$ such that $f=F_{1}+F_{2}+\cdots+F_{m}$, where $F_{i}=$ $f_{1}^{(i)} f_{2}^{(i)} \ldots f_{n_{i}}^{(i)}$ and $f_{j}^{(i)} \in \Lambda, j=1,2, \ldots, n_{i}, i=1,2, \ldots, m$. Let $N=$ $\max \left(n_{1}, n_{2}, \ldots, n_{m}\right)$. Since

$$
\sqrt[N]{|f|} \leqslant \sqrt[N]{\left|F_{1}\right|}+\sqrt[N]{\left|F_{2}\right|}+\cdots+\sqrt[N]{\left|F_{m}\right|}
$$

to establish (1) it suffices to demonstrate that

$$
\varlimsup_{y \rightarrow x} \frac{1}{|y-x|} \int_{x}^{y N} \sqrt{\left|F_{i}(t)\right|} d t<\infty, \quad i=1,2, \ldots, m .
$$

Define

$$
G_{i}=\max \left(\left|f_{1}^{(i)}\right|,\left|f_{2}^{(i)}\right|, \ldots,\left|f_{n_{i}}^{(i)}\right|, 1\right), \quad i=1,2, \ldots, m .
$$

Since $\left|f_{j}^{(i)}\right| \in \Lambda, j=1,2, \ldots, n_{i}$, it is evident that each $G_{i} \in \Lambda$. Thus,

$$
\lim _{y \rightarrow x} \frac{1}{|y-x|} \int_{x}^{y} G_{i}(t) d t<\infty
$$


for each $x \in[a, b]$. Observe that

$$
\left|F_{i}\right|=\left|f_{1}^{(i)}\right|\left|f_{2}^{(i)}\right| \ldots\left|f_{n_{i}}^{(i)}\right| \leqslant\left|G_{i}^{n_{i}}\right| \leqslant G_{i}^{N} .
$$

Hence, $\sqrt[N]{\left|F_{i}\right|} \leqslant G_{i}$, so (2) implies that for each $x$

$$
\varlimsup_{y \rightarrow x} \frac{1}{|y-x|} \int_{x}^{y N} \sqrt{\left|F_{i}(t)\right|} d t<\infty, \quad i=1,2, \ldots, m .
$$

This establishes necessity.

To prove sufficiency, suppose that $f \in C A$ and there exists a positive integer $N$ such that (1) is satisfied for all $x$. Without loss of generality we may assume that $N$ is odd. By the remark we made after Definition $3.2, \sqrt[N]{|f|}$ has no firm nonLebesgue points. Let $h$ be the odd homeomorphism in $\mathcal{H}$ defined by $h(u)=\sqrt[3]{u}, u$ $\in R$. Then, applying Theorem 3.4 to the function $F=\sqrt[N]{|f|}$, it follows that $h \circ F \in \Lambda$. But

$$
h \circ F=\sqrt[3 N]{|f|}=|\sqrt[3 N]{f}|
$$

which implies that $\sqrt[3 N]{f} \in \Lambda$. Since $f=(\sqrt[3 N]{f})^{3 N}, f$ is a product of $3 N$ functions in $\Lambda$. Thus $f \in \Lambda_{*}$, completing the proof.

Our closing theorem shows that in a certain sense the classes $\Lambda$ and $\Lambda_{*}$ are equivalent.

THEOREM 5.4. There exists a homeomorphism $h \in \mathcal{H}$ such that $h \circ f \in \Lambda$ for every $f$ in $\Lambda_{*}$.

PROof. For each odd positive integer $p$, define a homeomorphism $h_{p} \in \mathcal{H}$ by $h_{p}(u)=\sqrt[p]{u}$. By Lemma 3.6, there exists an odd homeomorphism $h \in \mathcal{H}$ such that for every $p$ there is a $j_{p}$ for which $|u|>j_{p}$ implies $|h(u)| \leqslant\left|h_{p}(u)\right|$. Let $f$ be any member of $\Lambda_{*}$. By using part of the previous proof, we conclude that there exists an odd positive integer $N$ such that $\sqrt[3 N]{f}$ belongs to $\Lambda$. But $\sqrt[3 N]{f}=h_{3 N} \circ f$, so $h_{3 N} \circ f \in \Lambda$. Since $|h(u)| \leqslant\left|h_{3 N}(u)\right|$ for all sufficiently large $|u|$, Lemma 3.3 implies that $h \circ f \in \Lambda$. This completes the proof.

The analogue to Theorem 5.4 does not hold for the class $C A^{*}$. In fact, if $h$ is any member of $\mathcal{K}$, then it is possible to construct a summable $f \in C A^{*}$ such that $h \circ f \notin \Lambda$. (Details may be found in [8].) This shows how much "larger" the class $C A^{*}$ is than the class $\Lambda_{*}$ which it contains.

\section{REFERENCES}

1. N. Bari, Mémoire sur la représentation finie des fonctions continue, Math. Ann. 103 (1930), 185-248.

2. A. M. Bruckner, Creating differentiability and destroying derivatives, Amer. Math. Monthly 85 (1978), 554-561.

3. Differentiability a.e. and approximate differentiability a.e., Proc. Amer. Math. Soc. 66 (1977), 294-298.

4. __ Differentiation of real functions, Lecture Notes in Math., vol. 659, Springer-Verlag, Berlin, 1978.

5. __ Inflexible derivatives, Quart. J. Math. Oxford Ser. (2) 29 (1978), 1-10. 
6. A. M. Bruckner and C. Goffman, Differentiability through changes of variable, Proc. Amer. Math. Soc. 61 (1976), 235-241.

7. R. J. Fleissner and J. Foran, Transformations of differentiable functions, Colloq. Math. (to appear).

8. D. Hancock, Homeomorphic transformations of approximately continuous functions into derivatives, Doctoral Dissertation, University of California, Santa Barbara, 1979.

9. M. Iosifescu, Conditions that the product of two derivatives be a derivative, Rev. Roumaine Math. Pures Appl. 4 (1959), 641-649.

10. I. Maximoff, On the continuous transformation of some functions into an ordinary derivative, Ann. Scuola Norm Sup. Pisa. 12 (1943), 147-160.

11. __ Sur la transformation continue de fonctions, Bull. Soc. Phys.-Math. Kazan (3) 12 (1940), 9-41.

12. Sur la transformation continue de quelques fonctions en dérivées exactes, Bull. Soc. Phys.-Math. Kazan (3) 12 (1940), 57-81.

13. I. Natanson, Theory of functions of a real variable, Vol. II, Ungar, New York, 1960.

14. D. Preiss, Maximoff's theorem, Real Anal. Exchange 5 (1980), 92-104.

Department of Mathematics, Pepperdine University, Malibu, Californi 90265 